621.851 .03

\title{
Safety Domain of Lateral Deflection of Yarn Running on Guide Pulleys*
}

\author{
By Toshihiro IrIE**, Gen YamadA***, and Masakatsu HaYAShI****
}

\begin{abstract}
Flexible stranded wire or yarn running on guide pulleys comes to leave groove surfaces of pulleys, when angles between wire (or yarn) and the central plane of pulleys become large.

In this paper, critical angles at which yarn is going to run off groove edge of test pulleys, and the effects of groove angle, number of pulleys, s'pporting condition of pulleys, running speed and tension of yarn on the critical angles, were studied experimentally by using cotton yarn and model pulley system consisting of a single, two or four wheels of V-type groove. Curves of critical angles of running yarn in the inlet and outlet of pulley system were found to be grouped into three types_- parallel, rectangular and complex type according to the number of pulleys and supporting condition. And a method of determining safety domain of lateral deflection of running yarn was proposed.
\end{abstract}

\section{Introduction}

Machines of the mechanism, in which flexible string or yarn runs on grooved pulleys, are used in many industrial fields. String or yarn does not always run in the central plane of grooved pulleys, but it sometimes runs deflecting from the central plane. Definite information and sufficient data on the safety for running off grooved pulleys have not been obtained, though it is an important problem from the viewpoints of design, operation and maintenance of machine.

In this paper, critical angles at which cotton yarn is going to run off the groove edge of test pulleys, as the result of yarn deflecting from the central plane, are measured and the effects of bent angle of yarn, groove angle, number of pulleys, supporting condition of pulleys, running speed and tension, on the critical angles, are studied experimentally by using cotton yarn and model pulleys system consisting of a single, two or four wheels of V-type groove. Curves of critical angle of running yarn at the inlet and outlet of pulleys system are classified into three typical forms and a method of determination of safety domain for lateral deflection

* Received 25 th April, 1968.

** Professor, Faculty of Engineering, Hokkaido University, Sapporo.

*** Assistant Professor, Faculty of Engineering, Hokkaido University.

**** Student, Graduate School of Engineering, Hokkaido University. of running yarn is proposed.

\section{Experimental apparatus and method}

Experimental apparatus consists of the five main parts-grooved test pulleys constructed with a single, two or four wheels (V), a guide pulley on the inlet side to deflect yarn $\left(\mathrm{P}_{1}\right)$, a guide pulley on the outlet side $\left(\mathrm{P}_{2}\right)$, a drive pulley to travel. yarn $\left(P_{d}\right)$, and a tension pulley to stretch yarn at a constant tension $\left(P_{t}\right)$, which are arranged horizontally as shown in Fig. 1. An example of test pulleys system (four wheels) is shown in Fig. 2; this system can move continuously in the vertical direction for changing arbitrarily the bent angle $\alpha$ of yarn on the inlet and outlet sides to test pulleys. The test pulleys system can rotate freely about the vertical axis by a bearing or be fixed rigidly at the vertical axis or can also be provided with an elastic spring in order that the restoring moment may act on the pulleys rotating from the neutral position. Multi-pulleys system is also free to rotate around the horizontal axis by a pin $Q$. Two guide pulleys can move on the horizontal table in the longitudinal direction of experimental apparatus, so that guide pulleys distances $L_{1}, L_{2}$ (horizontal distances between the shaft of test pulleys and guide pulley) are set up arbitrarily. And the guide pulleys also move horizontally in the lateral direction of the apparatus, so that the deflection angles $\varphi_{1}$ and $\varphi_{2}$ of yarn relative to test pulleys can be changed continuously. The angular velocity of a drive pulley is 
changed by a stepless speed change device and, therefore, running speed $U$ of yarn is varied. For fixing the tension $T$ of endless yarn, even when guide pulley moves to deflect yarn, a counter weight and tension pulley movable in the longitudinal direction are provided. Subscripts 1 and 2 of the notations in this paper are used to distinguish the quantities on the inlet and on the outlet side of yarn.

Using the above-mentioned device, the distance of the guide pulley is changed arbitrarily by the movement of guide pulley, the bent angle of yarn by the vertical movement of test pulleys and the tension of yarn by a stretching device respectively. And critical deflection angles of running yarn, at which yarn is going to run off the groove edge of test pulleys, are measured when one of the guide pulleys is moved in the lateral direction perpendicular to yarn, fixing the other pulleys.

In this paper, light cotton thread $(0.85 \mathrm{~g} / \mathrm{m})$
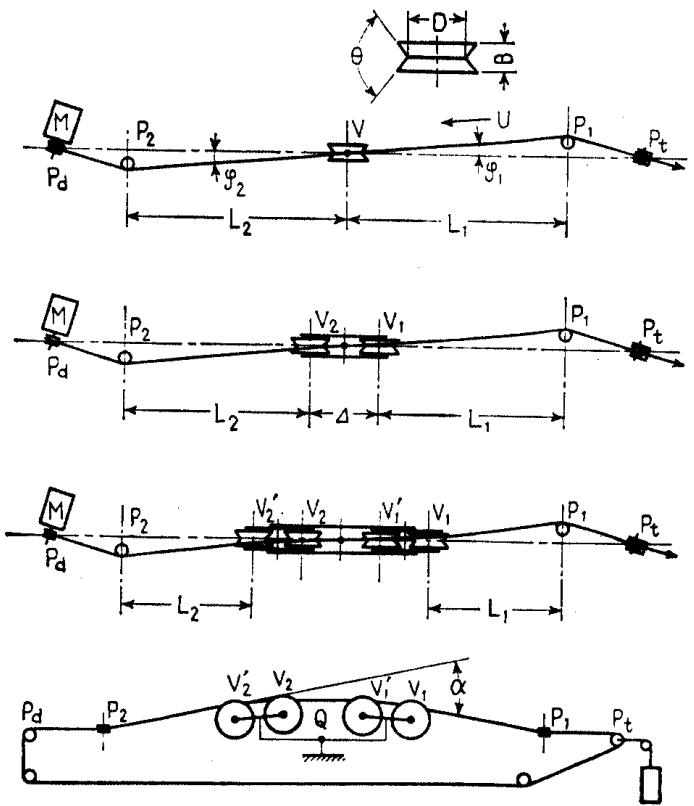

Fig. 1 Arrangement of Dulters

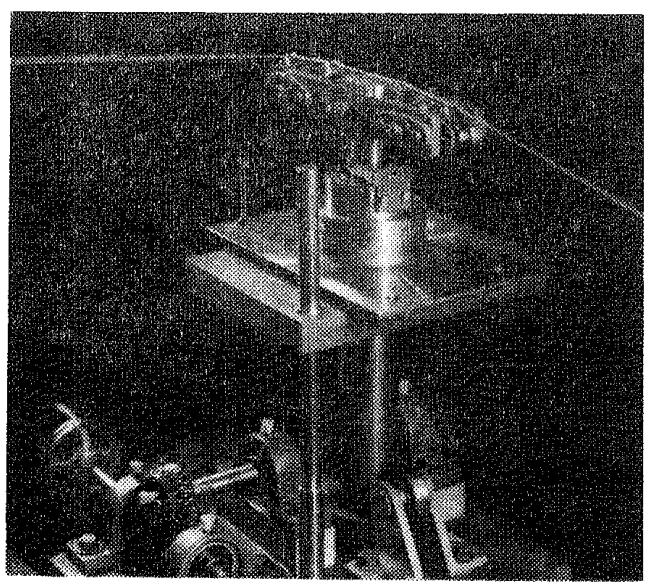

Fig. 2 Supporting device of test pulleys (four pulleys) was used as test yarn, and mild steel pulleys of Vtype groove, the surface of which was treated by high-frequency hardening and which had the following dimensions :

Diameter of the groove bottom of pulleys: $D$ $=40 \mathrm{~mm}$

Breadth of pulleys : $b=B / D=0.15,0.45,0.60$, 0.75

Groove angle of pulleys : $\theta=60^{\circ}, 75^{\circ}, 90^{\circ}$

Distance between two test pulleys: $d=\Delta / D$ $=1 \frac{1}{2}, 2,3,4$

were adopted as test pulleys. Guide pulleys and tension pulley were also made of mild steel of $\mathrm{V}$. type groove and drive pulley was made of vinyl chloride. The distance between test pulleys and guide pulley was $\left(l_{1}, l_{2}\right)=\left(L_{1}, / D, L_{2} / D\right)=15 \sim 100$, running speed of yarn $U=0.1 \sim 0.9 \mathrm{~m} / \mathrm{sec}$, tension of yarn $T=0.10 \sim 0.60 \mathrm{~kg}$, rotary spring stiffness about the vertical shaft of test pulleys $k_{\mathrm{t}}=0$ (free), 0.21 , $1.69 \mathrm{~g} \cdot \mathrm{mm} / \mathrm{deg}, \infty$ (fixed). As the tensile strength of test yarn was about $10 \mathrm{~kg}$, the ratio of tension of yarn to the tensile strength was merely within the limits of $1 / 100 \sim 1 / 16$. Since the critical domain was seen to have a tendency to converge as tension of yarn increased, no experiments were carried out under large tension. The coefficient of dynamical friction between yarn and test pulleys is usually related with dimensions, materials of yarn and test pulleys, and many uncontrollable factors such as room temperature and humidity, and therefore, the values obtained experimentally should have considera. ble randomness. In order to determine the coefficient of dynamical friction, test yarn attaching two masses $m_{1}, m_{2}\left(m_{1}>m_{2}\right)$ to its both ends, is hung on a rod of circular cross section, $15 \mathrm{~mm}$ in dia., which is made of the same material as test pulleys and is fixed horizontally, and then the accerelation $\alpha$ when yarn slides toward the side of large mass is measured. The coefficient of dynamical friction of yarn is obtained by eliminating the tension $T_{1}, T_{2}$ from the equations

$$
\begin{aligned}
& m_{1} \alpha=m_{1} g-T_{1},-m_{2} \alpha=m_{2} g-T_{2}, \\
& T_{1}=T_{2} e^{\mu \theta} \quad\left(\theta=180^{\circ}\right)
\end{aligned}
$$

Therefore,

$$
\mu=\frac{1}{\pi} \log _{e}\left(\frac{m_{1}}{m_{2}} \frac{1-\alpha / g}{1+\alpha / g}\right)
$$

According to the experimental results, the value of $\mu$ was in the vicinity of 0.25 .

\section{Experimental results and the considerations}

From the experimental results thus obtained, critical deflection angle of yarn and the effects of the supporting condition of pulleys on the critical 
angle are discussed here in detail and compared with each other.

\subsection{A single pulley fixed at the vertical axis}

Some curves of critical angle of yarn are presented in Figs. 4, 6 8, in which the deflection angle $\varphi_{1}$ at the inlet is taken as abscissa and the angle $\varphi_{2}$ at the outlet as ordinate. If the deflection angles on both sides stay within these curves, yarn continues to run on grooved pulley, but when the angle reaches the critical angle on the curves, yarn is going to run off the groove edge.

Only the upper half of curves of critical angle (I and II quadrant) is shown in Figs. 4 and 7, omitting complete curves, because of the symmetricity of the position of yarn to pulleys as seen in Fig. 3. Strictly speaking, this symmetricity was lost a little owing to twist and rotation of yarn, but their influences on cotton yarn were not considered to be so serious in this experiment. Curves of critical angle in I and III quadrants, correspond to the case when yarn is on the opposite side of the central plane of test pulleys, and curves in II and IV quadrants correspond to the case when yarn is on the same side as seen in Fig. 3. In I (III) quadrant of Fig. 4 , there are two critical angles and this is explained from the fact that on the outside (top-left) of the upper critical angles, yarn goes to run off the groove at the outlet, and on the outside (bottom. right) of the lower critical angles, yarn runs off on

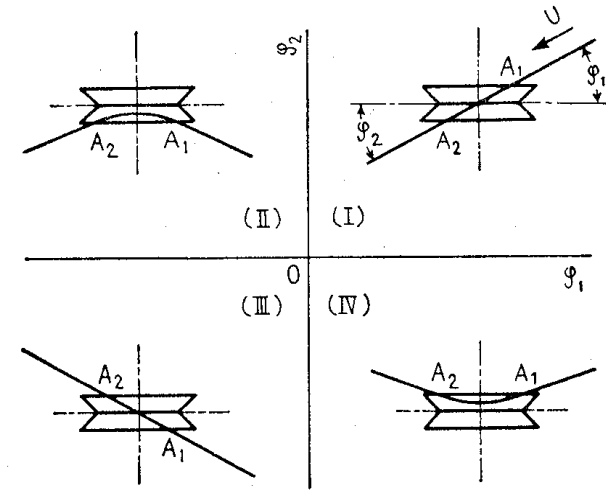

Fig. 3 Relative position of yarn to test pulleys

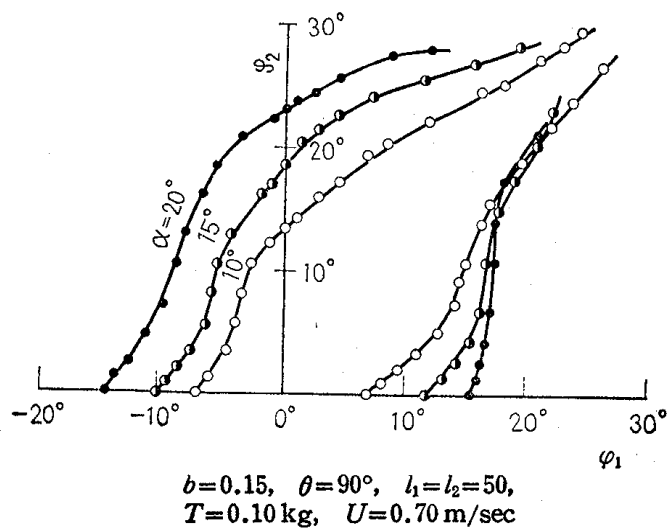

Fig. 4 Critical angles for bent angle (a single pulley) the inlet side. As the deflection angles on both sides become large, yarn rises gradually to the groove edge, contacting on a line with the surface, and at last comes to contact with only two points $A_{1}, A_{2}$ of the pulley as seen in Fig. 3. And it becomes impossible to continue the experiments and the bent angle comes to differ from what is obtained in this paper.

It is found from Fig. 4, that critical domain comes to expand as the bent angle of yarn increases, and this will be explained from the fact that when the deflection increases, the length of contact line between yarn and groove surface becomes long and the restoring force to put yarn back to the bottom of groove becomes large.

In II (and IV) quadrant of these curves where yarn is on the same side of the central plane of test pulleys, critical domain decreases in general. In this case, the critical angles at the inlet and outlet $\left|\varphi_{1}\right|_{0}$ and $\left|\varphi_{2}\right|_{0}$, when the deflection angles at the outlet and inlet are set zero respectively, are shown in Fig. 5, taking the bent angle of yarn as abscissa. These critical angles increase almost linearly with an increasing bent angle.

Curves of critical angle of test pulleys for various breadths are shown in Fig. 6, where yarn contacts with the groove surface on only a single side of pulley and, therefore, critical domain is small. And remarkable effects of the breadth of test pulleys on the critical domain are little seen here. Figure 7 shows curves of critical angle of test pulleys for various groove angles, from which it is seen that critical domain is enlarged, as the groove angle becomes small, because the inclination of groove surface increases. And it is also seen in Fig. 8 that there are little effects of the distance between

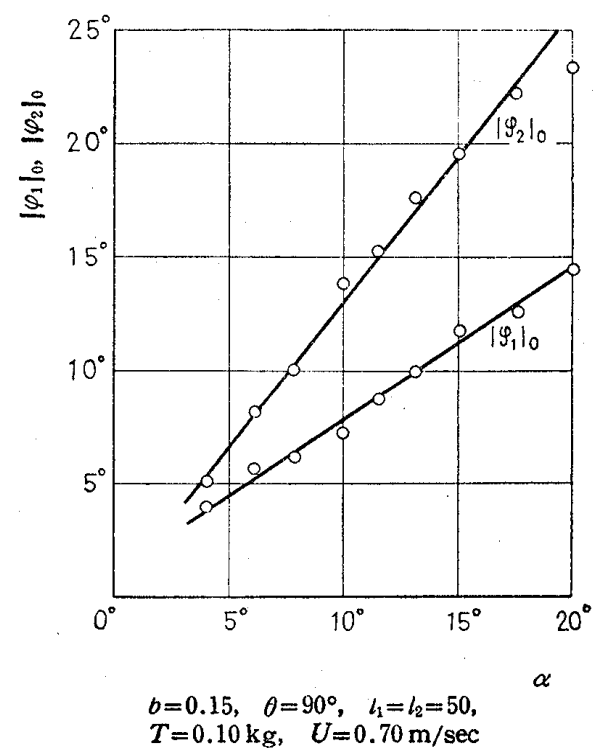

Fig. 5 Bent angle and critical angle (a single pulley) 
test pulleys and guide pulleys on the inlet and outlet side on the critical angles.

It will be found from these curves that the ratio of the inlet-side angle to the outlet-side one $\left|\varphi_{1}\right|_{0} /\left|\varphi_{2}\right|_{0}$ is almost $1 / 2 \sim 1 / 2.5$. And therefore, critical domain is affected remarkably by the inletside angle, so that special attention should be paid to this angle. It will be clear from the figures obtained later, that the $\left|\varphi_{1}\right|_{0} /\left|\varphi_{2}\right|_{0}$ of multi-pulleys (fixed) is also in the same level as that of a single pulley. In wirerope or wire with flexural rigidity, it is considered that though the relative position of a guide pulley to test pulleys is equal to each other, the contact length with groove surface becomes short, so that there is a tendency to run off the groove edge more easily than flexural cotton yarn.

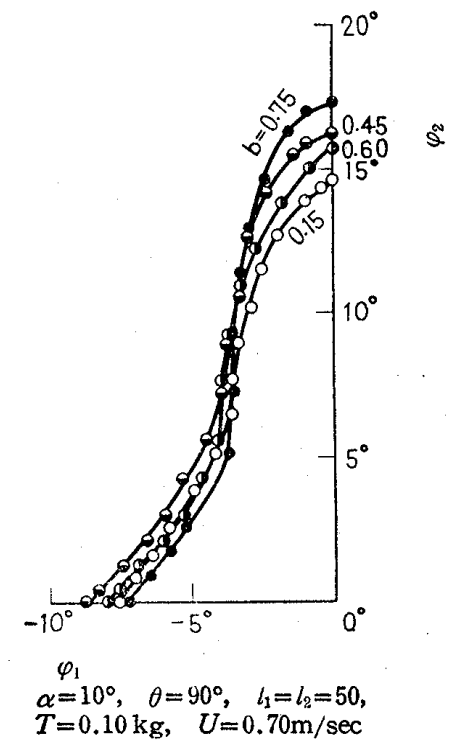

Fig. 6 Critical angles for breadth (a single pulley)
However, it was difficult to measure the critical angle of even the wirerope whose diameter was small $(3 \sim 4 \mathrm{~mm}$ ), by means of the small sizedapparatus used in this experiment.

\subsection{Two pulleys fixed at the vertical axis}

Curves of critical angles of two pulleys fixed at the vertical axis are shown in Figs. 9 11, where the curves are seen to be more sharp and hysterisislike than those of a single pulley.

Curves of critical angles for some different bent angles are shown in Fig. 9, where the bent angle of two pulleys system is represented by the sum of angle of each pulley and therefore, the bent angle per one pulley is equal to one-half that of a single pulley. Critical angle of yarn deflected on a single side increases almost linearly. in the same way as a single pulley, when the bent angle increases. As groove angle decreases, critical angle increases, as seen in Fig. 10, and hence, the danger of running off the pulley is reduced. Figure 11 shows the

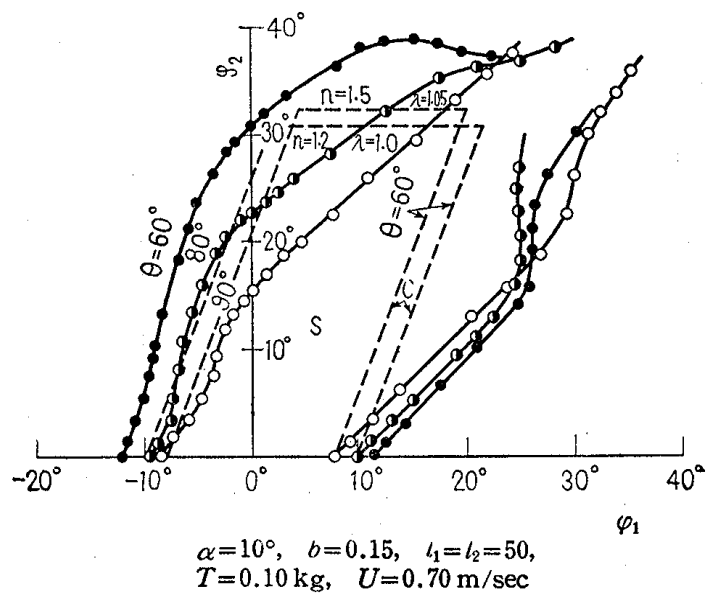

Fig. 7 Critical angles for groove angle (a single pulley)

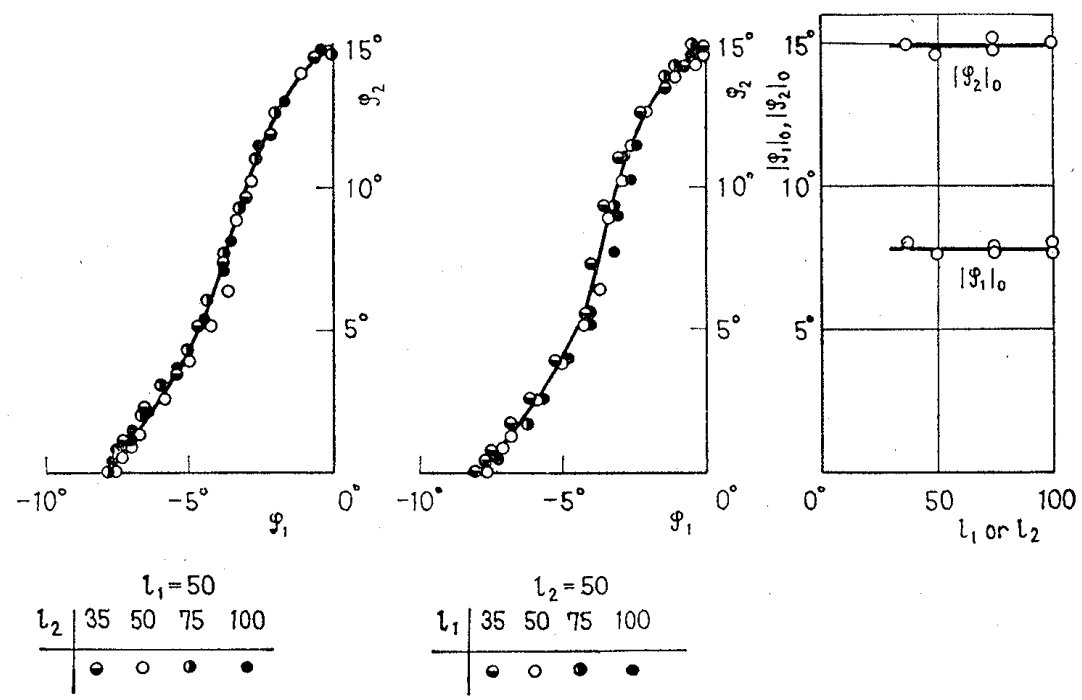

$\alpha=10^{\circ}, \quad b=0.15, \quad \theta=90^{\circ}, T=0.10 \mathrm{~kg}, \quad U=0.70 \mathrm{~m} / \mathrm{sec}$

Fig. 8 Critical angles for distance between test pulleys and guide pulleys (a single pulley) 
curves of test pulleys for various distances between the shafts of two pulleys, where critical domain is seen to decrease, as the distance becomes large.

\subsection{Four pulleys fixed at the vertical axis}

In Figs. 12 and 13, curves of critical angle in only I quadrant are shown, to which the curves in the other quadrants are almost symmetric. Figure 12 shows the curves of test pulleys for various bent angles, where the bent angle of four pulleys system is represented by a sum of that of each pulley. Since each test pulley is constructed to change freely the relative position in the central plane as shown in Fig. 1, the bent angle per one pulley is nearly equal to one-quarter of the total angle. The critical domain becomes large in the same way as a single or two pulleys, when the bent angle increases. And it is seen from Fig. 13, that the critical domain also enlarges, as the groove angle becomes small. It seems that the above mentioned tendencies for four pulleys system fixed at the vertical axis also hold good for the multi-pulleys system.

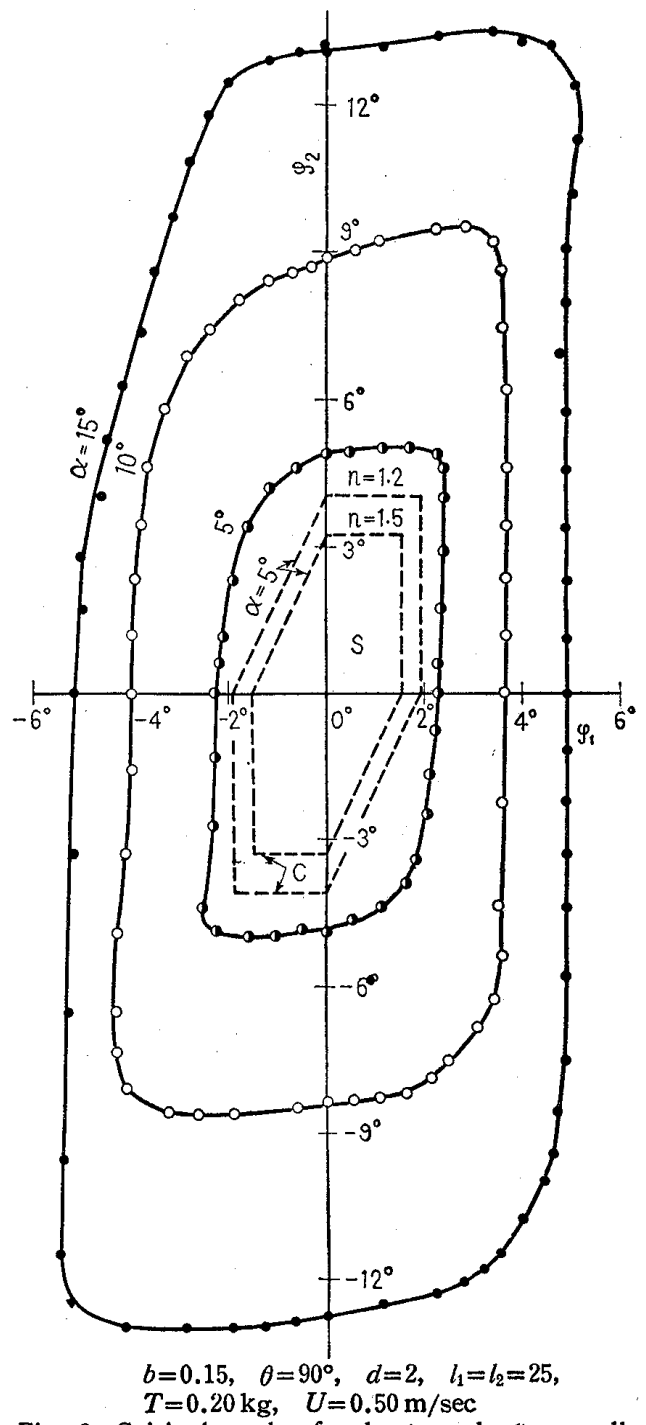

Fig. 9 Critical angles for bent angle (two pulleys)
The influences of tension and running speed of yarn on critical angle will be discussed, by taking. a four pulleys system as an example. The influences. of tension of yarn on the critical angle are shown in Fig. 14, from which it is seen that the critical angle at the inlet is little affected by the tension within the limits in the experiment and the angle at the outlet is almost constant, except under extremely small tension. The critical angle comes to increase a little, as the tension becomes small, but when the tension is extremely small, yarn is deflected by the gravity and the bent angle comes to differ from the original angle, and hence, such cases. are omitted in this experiment. There is a little difference between the tension of yarn on inlet and outlet side, but since the relative difference of tension is smaller than several percent within the limits. of the bent angle in this experiment, the magnitude

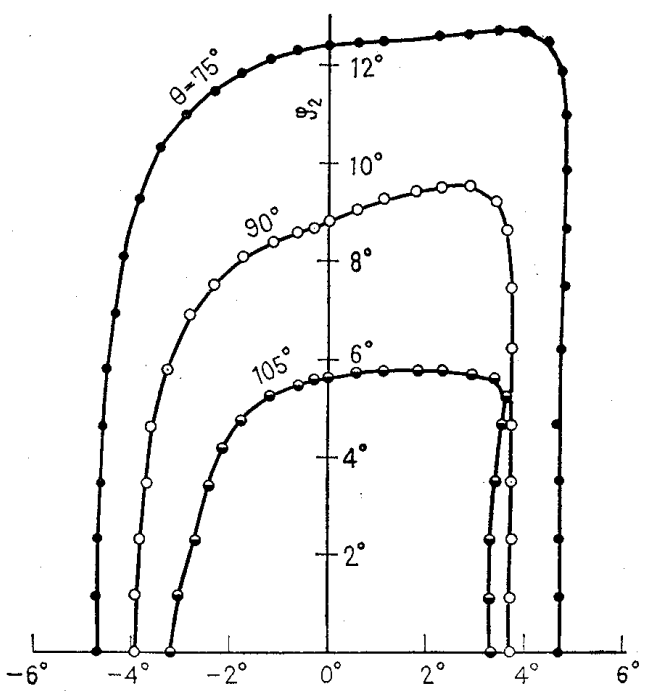

$$
\alpha=10^{\circ}, \quad \mathrm{b}=0.15, \quad d=2, \quad l_{1}=l_{2}=25,
$$$$
T=0.20 \mathrm{~kg}, \quad U=0.50 \mathrm{~m} / \mathrm{sec}
$$

Fig. 10 Critical angles for groove angle (two pulleys)

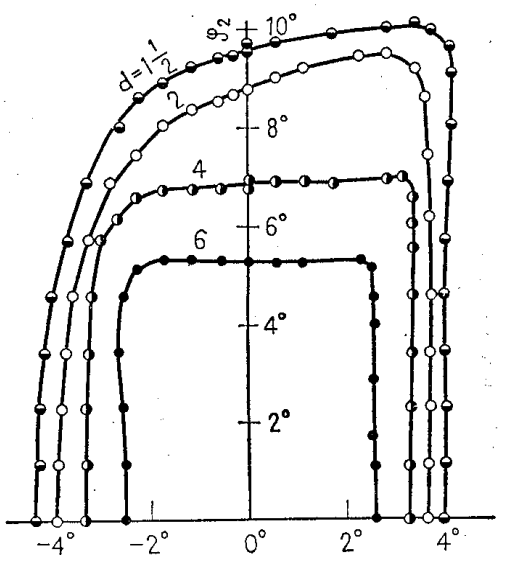

$\alpha=10^{\circ}, \quad b=0.15, \quad \theta=90^{\circ}, \quad l_{1}=l_{2}=25$, $T=0.20 \mathrm{~kg}, \quad U=0.50 \mathrm{~m} / \mathrm{sec}$

Fig. 11 Critical angles for distance between two test pulleys (two pulleys) 


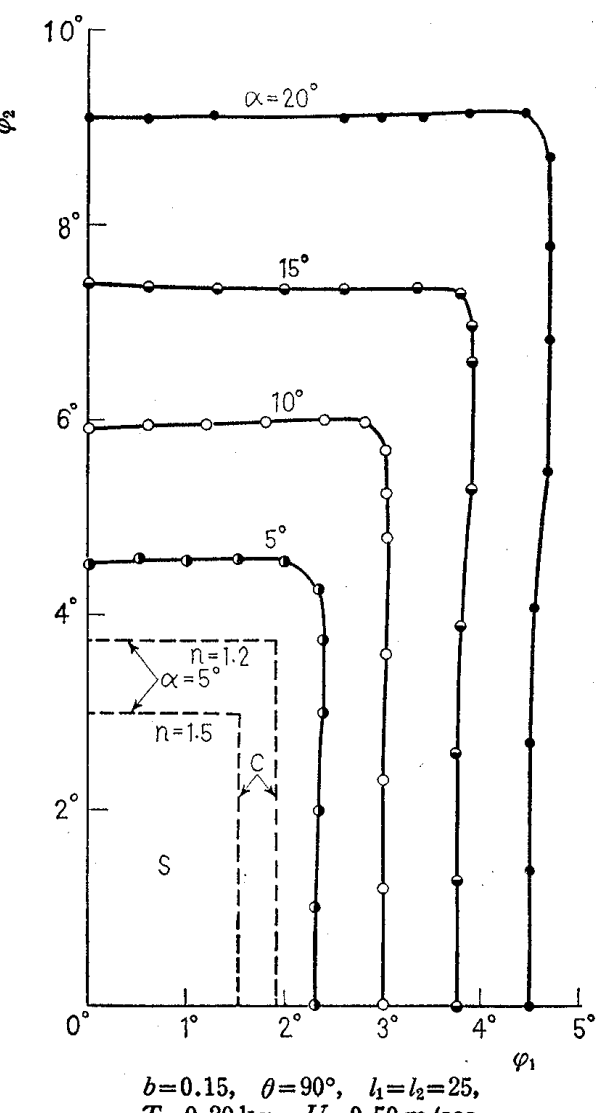

$b=0.15, \quad \theta=90^{\circ}, \quad l_{1}=l_{2}=25$,
$T=0.20 \mathrm{~kg}, \quad U=0.50 \mathrm{~m} / \mathrm{sec}$

Fig. 12 Critical angles for bent angle (four pulleys)

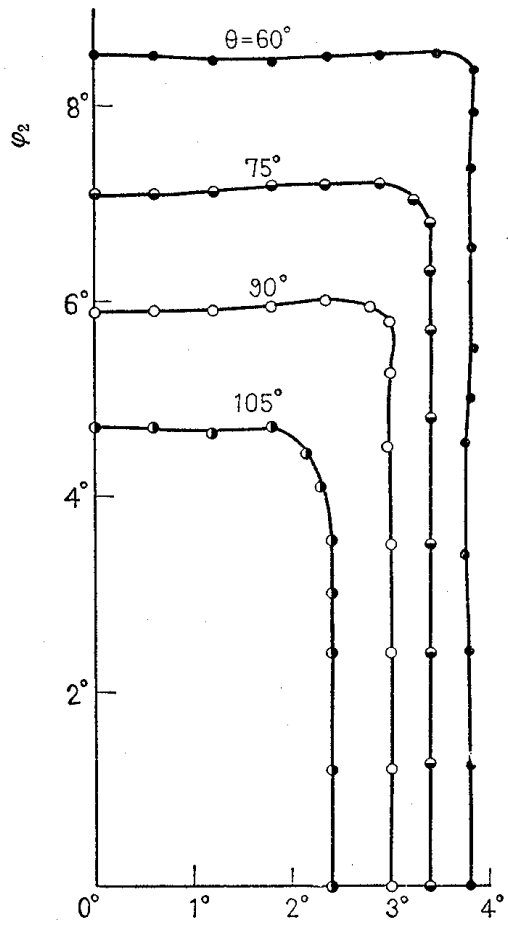

$\alpha=10^{\circ}, \quad b=0.15, \quad l_{1}=l_{2}=25$,

$T=0.20 \mathrm{~kg}, \quad U=0.50 \mathrm{~m} / \mathrm{sec}$

Fig. 13 Critical angles for groove angle (four pulleys) of tension on the outlet side tentatively is used here.

The influence of running speed of yarn is shown in Fig. 15, from which it is easily seen that critical domain of the angle on the inlet side is little affected, but critical angle on the outlet side in. creases almost linearly with the increasing of running speed. This tendency remains unchanged in a single or two pulleys system. When the running speed of yarn reaches a certain level, yarn begins to vibrate and then it will affect the critical angle, but the experiments are carried out only at the speed, when yarn never vibrates, and therefore no influences of vibration are considered here.

3.4 Test pulleys on which an elastically restoring moment acts

In the preceding sections, all test pulleys have been fixed at the vertical axis. The effects of a restoring moment acting on the test pulleys by an elastic spring about its vertical axis, will be studied here, and compared with the results obtained in the pulleys free-rotatable or fixed.

In Fig. 16, it is shown that, if the restoring moment is large, the critical domain is reduced and

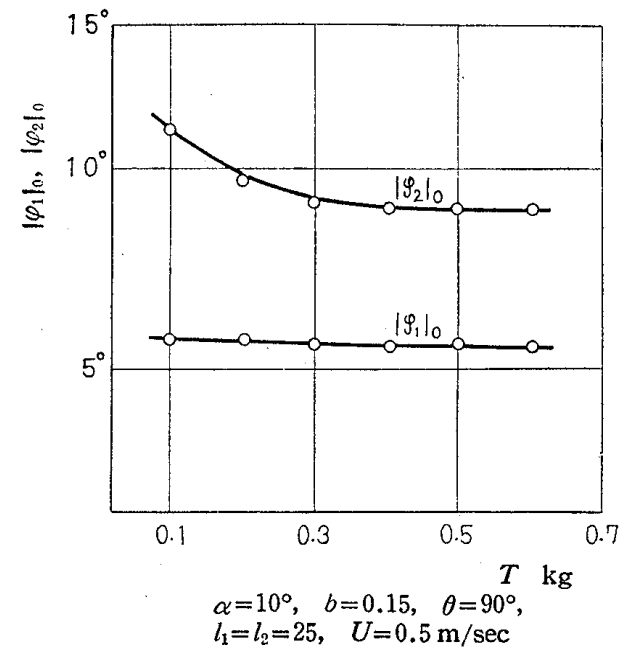

Fig. 14 Tension and critical angles (four pulleys)

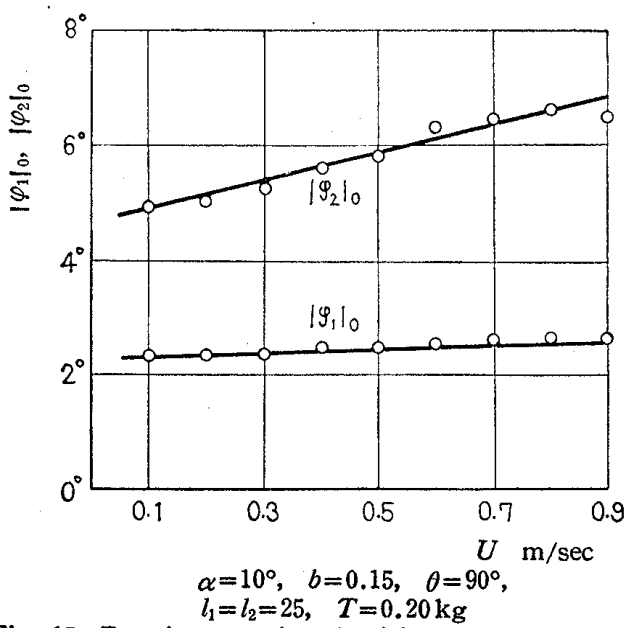

Fig. 15 Running speed and critical angles (four pulleys) 
the difference between the critical angles on both sides has a tendency to become small, as the moment decreases. The reason for this is that since test pulleys are constructed so as to rotate freely about the vertical axis, when the deflection angle of yarn on either side becomes large, the direction of test pulleys is changed by rotating about the vertical axis and the critical angle on the other side also increases, in I or III quadrant where yarn intersects the central plane of test pulleys. Therefore, in the case when test pulleys rotate freely, the curves of critical angle have a tendency to become almost linear and both of the critical angles on the inlet and outlet side become nearly equal to each other. As the rotary spring stiffness becomes large, the angular displacement of test pulleys about the vertical axis becomes small, and hence, the critical domain becomes a closed curve.

When the lateral vibration of yarn is transmitted to test pulleys and is out of phase with the rotation of test pulleys about the vertical axis, the

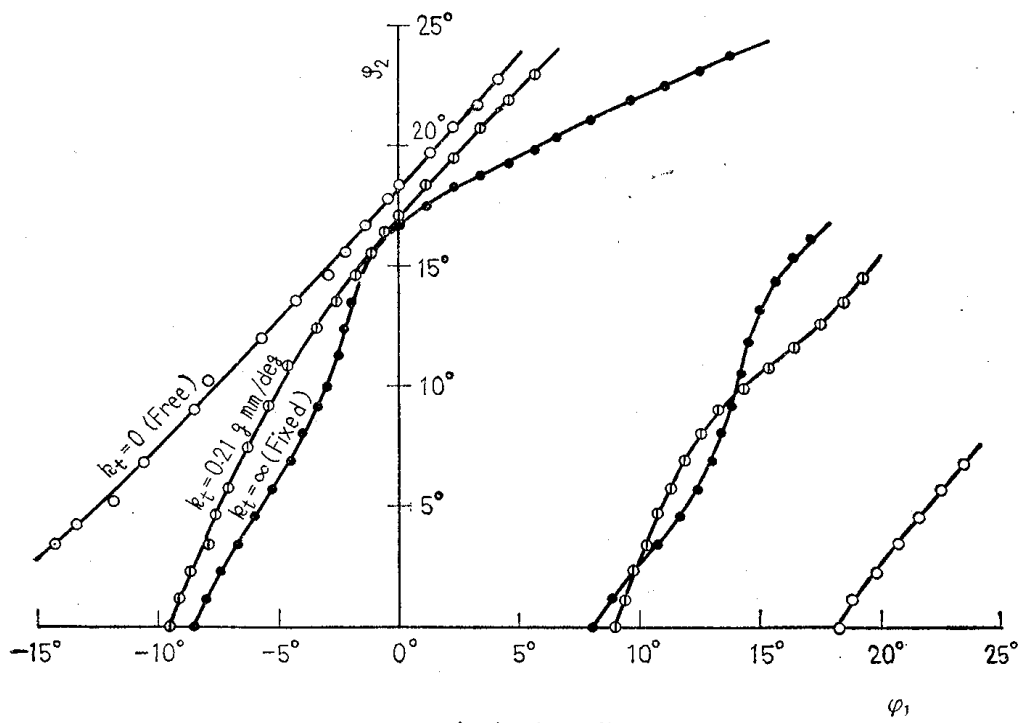

(a) A single pulley

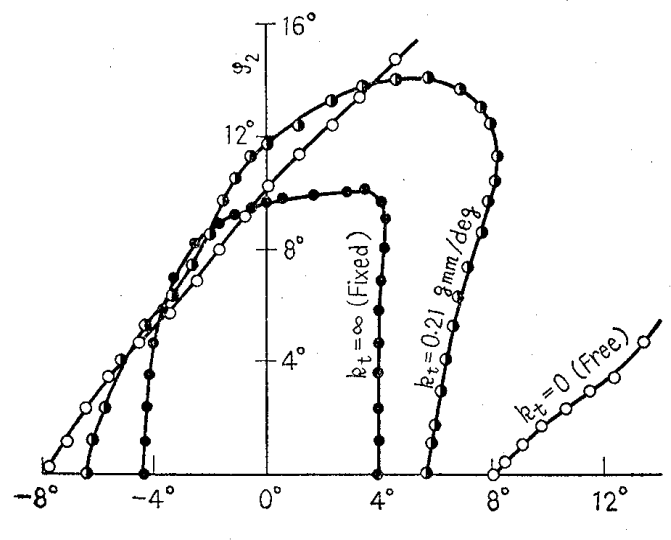

(b) Two pulleys

$\varphi_{1}$ dumping. wheels deflection angles on both sides become large, so that the danger of running off grooved pulley appears. It is considered more desirable that the restoring moment acting on the pulley is small for enlarging the critical domain; however, in practice, undesirable phenomena such as the turning of pulleys, the resonance with yarn, etc., are liable to occur, and therefore, it is desirable for test pulleys to be provided with a proper restoring moment and rotary

3.5 Comparison of critical domain of test pulleys constructed with a single, two or four

Attempt will be made to compare the test pulleys system consisting of a single, two or four pulleys with each other. As mentioned above, the bent angle of multi-pulleys has been defined by a sum of angle of each pulley, but when yarn runs off two pulleys, it occurs usually on either one of them, and in the case of four pulleys it occurs on one of the most outside pulleys. Therefore, for comparing these domains, it will be better to equalize the bent angle of test pulleys from which yarn runs off. In Fig. 17, curves of critical angle, of a single pulley whose bent angle is $5^{\circ}$, two pulleys whose angle is $10^{\circ}$, four pulleys whose angle is $20^{\circ}$, are compared with each other, in such

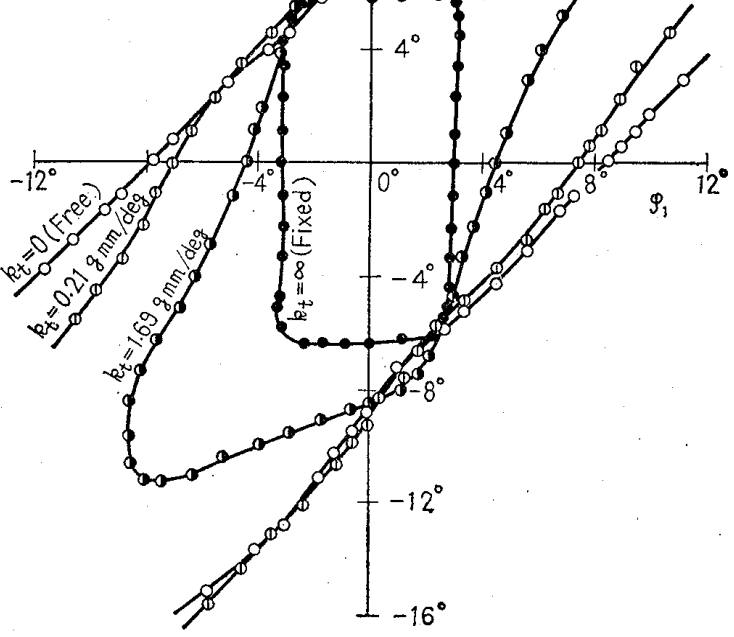

(c) Four pulleys

$\alpha=10^{\circ}, \quad b=0.15, \quad \theta=90^{\circ}, \quad l_{1}=l_{2}=25, \quad T=0.20 \mathrm{~kg}, \quad U=0.50 \mathrm{~m} / \mathrm{sec}$

Fig. 16 Critical angles of test pulleys on which elastically restoring moment acts 
manner that the bent angle per one pulley is always $5^{\circ}$.

Figures 17 (a) and (b) show curves of critical angle of test pulleys fixed and free-rotatable about its axis respectively. Curves of critical angle of fixed pulleys approach a rectangular type and hence the critical domain enlarges, as the number of pulleys increases. The reason is that, as the number of pulleys increases, one of the most outside pulley going to run off is little affected by the other most outside pulley and keeps a large critical angle, tending to $\left|\varphi_{1}\right| \approx\left|\varphi_{1}\right|_{0},\left|\varphi_{2}\right| \approx\left|\varphi_{2}\right|_{0}$, without regard to the deflection angle on the other side.

\section{Classification of critical curves and safety domain}

Critical domain on the running off of yarn has been discussed considerably in detail and here, a method for determination of safety domain will be proposed, by arranging the results. As seen in Fig. 18 and Table 1, the curves of critical angle of yarn will be classified into three types- (a) parallel type, (b) complex type and (c) rectangular type. In parallel type, when yarn is on the opposite side of the central plane of pulleys (I and III quadrants),
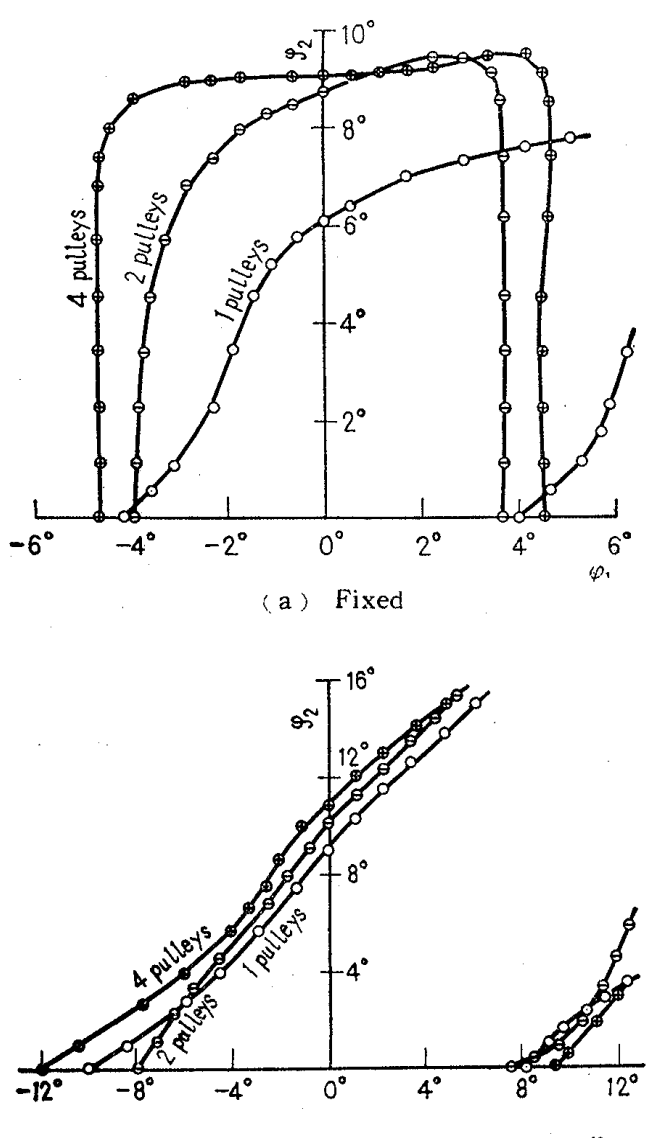

(b) Free

$\varphi_{1}$

$\alpha=5^{\circ}$ (per a single pulley), $b=0.15$,

$\theta=90^{\circ}, \quad l_{1}=l_{2}=25, \quad T=0.20 \mathrm{~kg}, \quad U=0.50 \mathrm{~m} / \mathrm{sec}$

Fig. 17 Critical angles for the number of pulleys

Table 1 Type of curves of critical angle

\begin{tabular}{|c|c|c|c|}
\hline Type & (a) Parallel type & (b) Complex type & $\left.\right|^{(c) \underset{\text { Rype }}{\text { Rectangular }}}$ \\
\hline Free & $\begin{array}{l}1,2,4 \text { pulleys : Free } \\
\text { Figs. } 16(\mathrm{a}) \text {, } \\
\text { (b), (c), 17(b) }\end{array}$ & & \\
\hline \multirow{3}{*}{ Elastic } & $\begin{array}{l}1 \text { pulley : Elastic } \\
\text { Fig. } 16(\mathrm{a})\end{array}$ & & \\
\hline & $\begin{array}{l}2 \text { pulleys : Elastic } \\
\text { Fig. } 16 \text { (b) }\end{array}$ & $\longrightarrow$ Large & \\
\hline & $\begin{array}{r}4 \text { (multi-) pulleys } \\
\text { : Elast } \\
\text { Fig. } 16 \text { (c) }\end{array}$ & tic & $\longrightarrow$ Large \\
\hline Fixed & $\begin{array}{l}1 \text { pulley: Fixed } \\
\text { Figs. 4, } 7 \\
17 \text { (a) }\end{array}$ & $\begin{array}{l}2 \text { pulleys: Fixed } \\
\text { Figs. } 9,10,11, \\
17 \text { (a) }\end{array}$ & $\begin{array}{l}4 \text { (multi-) pulleys } \\
\text { : Fixed } \\
\text { Figs. 12, 13, } \\
17 \text { (a) }\end{array}$ \\
\hline \multicolumn{4}{|c|}{ Table 2 Safety domain } \\
\hline Type & (a) Parallel type & (b) Complex type & $e^{(c) \operatorname{Rectangular}_{\text {type }}}$ \\
\hline $\begin{array}{l}\text { Safety } \\
\text { domain }\end{array}$ & $\left\{\begin{aligned} \varphi_{2}= & \pm \lambda\left|\varphi_{2}\right|_{0} \\
\varphi_{2} & =\frac{\left|\varphi_{2}\right|_{0}}{\left|\varphi_{1}\right|_{0}} \\
& \times\left(\varphi_{1} \pm \frac{1}{n}\left|\varphi_{1}\right|_{0}\right)\end{aligned}\right.$ & $\begin{aligned} \varphi_{1}= & \pm \frac{1}{n}\left|\varphi_{1}\right|_{0} \\
\varphi_{2}= & \pm \frac{1}{n}\left|\varphi_{2}\right|_{0} \\
\varphi_{2}= & \frac{\left|\varphi_{2}\right|_{0}}{\left|\varphi_{1}\right|_{0}} \\
& \times\left(\varphi_{1} \pm \frac{1}{n}\left|\varphi_{1}\right|_{0}\right)\end{aligned}$ & $\left\{\begin{array}{l}\varphi_{1}= \pm \frac{1}{n}\left|\varphi_{1}\right|_{0} \\
\varphi_{2}= \pm \frac{1}{n}\left|\varphi_{2}\right|_{0}\end{array}\right.$ \\
\hline
\end{tabular}

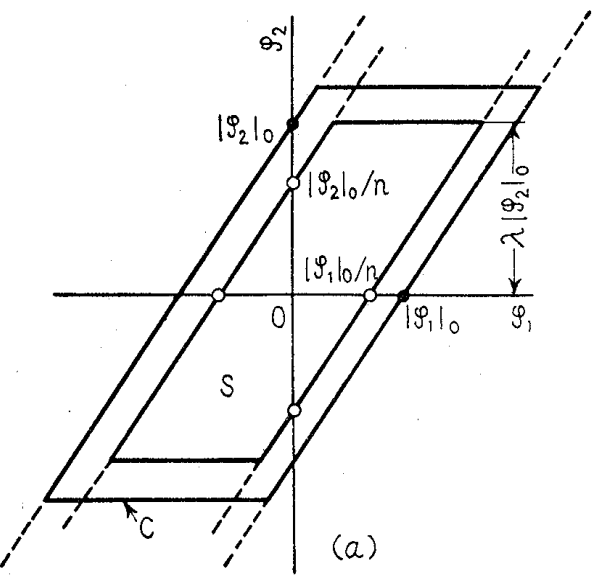

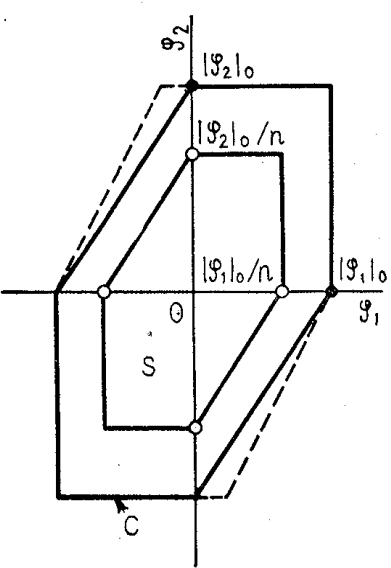

(b)

Fig. 18 Classification of critical curves and safety domain

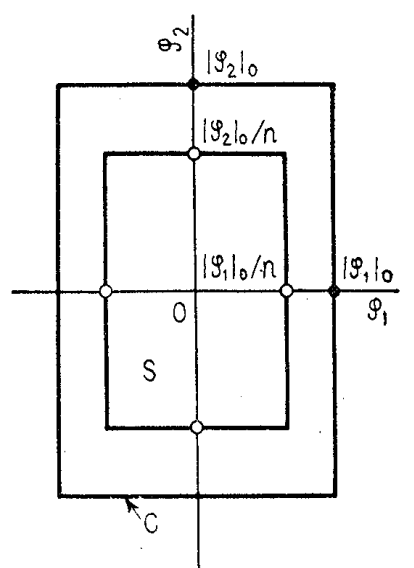

(c) 
two critical curves in the top-left and bottom-right part are nearly parallel; and they are closed in the upper (or lower) part, or they extend in parallel with each other till large deflection angle. And curves of this type occur in test pulleys which can rotate freely or in a single fixed pulley. In complex type, the critical curves are closed in the same way as rectangular type, when yarn is on the opposite side of the central plane of test pulleys; the curves are closed like a triangle (or trapezoid) when yarn is on the same side (II, IV quadrant). Curves of this type are seen in the case of two pulleys fixed at the axis. Curves of rectangular type are all closed like a rectangle and are seen in four or multi-pulleys fixed at the vertical axis. As shown in Table 1, test pulleys whose rotation is elastically constrained, have a tendency to change from parallel type to rectangular type, when the restoring moment becomes large.

For the above mentioned types, it is proposed by the authors that an area which is enclosed by some simple straight lines represented in Table 2 (see also Fig. 18), should be introduced as safety domain where yarn is guaranteed to run on the pulleys. Examples of the safety domain of parallel, complex and rectangular types are shown in Figs. 7,9 and 12 respectively.

The angles $\left|\varphi_{1}\right|_{0},\left|\varphi_{2}\right|_{0}$ and parameters $n$ and $\lambda$ are concerned with a determination of the safety domain, where $\left|\varphi_{1}\right|_{0}$ and $\left|\varphi_{2}\right|_{0}$ are critical angles on one side, when the deflection angles on the other side are zero, and are easily measured. Also $n$ $(>1)$ is a safety factor for deflection angle of yarn on a single side and the factor $\lambda(\geqq 1)$ determining the critical safety lines of parallel type is generally decided by the bent angle, groove angle and safety factor and the like. According to these experiments, the factor $\lambda$ may take a small value as the bent angle and groove angle increase, but it should be reasonably estimated with the safety factor, considering the character of machine. If sufficient experimental data have been obtained, it will be expected that the safety factor can be taken smaller to some extent; the factor $\lambda$ can be taken great, and therefore, a large safety domain can be adopted.

\section{Conclusions}

From the experimental results, the conclusions on the lateral deflection are reached as follows.

(1) Grooved pulleys fixed at the vertical axis:

(a) Bent angle: In a single, two or four pulleys, critical domain is enlarged, as bent angle becomes large.

(b) Breadth of grooved pulley: Critical domain is little affected by the breadth.

(c) Groove angle: Critical domain is enlarg. ed, as the angle becomes small.

(d) Distance of guide pulley: Critical domain is hardly affected by the distance.

(e) Tension of yarn: Except extremely small tension, critical domain is influenced little by tension, without regard to the number of pulleys.

(f) Running speed of yarn: This has hardly any influence on the deflection angle at the inlet, but critical angle on the outlet side tends to increase as the speed increases.

(2) Grooved pulleys on which an elastically restoring moment acts: The smaller the restoring moment, the larger the critical domain.

(3) Comparison of a single, two or four pulleys systems: As the number of pulleys increases, the critical domain is enlarged.

(4) A proposal of safety domain:

It is proposed that the curves' of critical angle of yarn are classified into three types and the safety domain of each type may be represented by some simple straight lines, and some examples are presented.

\section{Acknowledgement}

A part of this research was supported by THE SAKKOKAI FOUNDATION and one of the authors, G. YAMADA, wishes to thank for their support.

\section{References}

(1) T. Irie et al.: Preprint of 11 th Lecture Meeting Hokkaido-branch, Japan Soc. Mech. Engrs., (1966), p. 205.

(2) T. Irie et al.: Preprint of Japan Soc. Mech. Engrs., No. 173 (1967), p. 131. 